\title{
Simulation of ion transport in an extended electron cyclotron resonance plasma
}

\author{
Minghai Liu ${ }^{\text {a) }}$ and Xiwei Hu \\ Department of Modern Physics, University of Science and Technology of China, Hefei, Anhui, 230026, \\ People's Republic of China \\ Hanming Wu \\ Institute of Mechanics, Academia Sinica, Beijing, 100080, People's Republic of China \\ Qinchong Wu, Guoyang Yu, and Zhaoxing Ren \\ Institute of Plasma Physics, Academia Sinica, Hefei, Anhui, 230031, People's Republic of China
}

(Received 21 July 1998; accepted for publication 26 October 1999)

\begin{abstract}
A two-dimensional hybrid model for an electron cyclotron resonance (ECR) plasma reactor is improved by taking into account the microwave absorption. With this code, the velocity and angle distribution function of ions in the source and downstream areas, and the ion current density at the substrate surface in an extended ECR plasma reactor were investigated. The spatial profiles as well as the dependence of these distribution functions on pressure and microwave power were discussed. It is shown that the results are in good agreement with many experimental measurements. (C) 2000 American Institute of Physics. [S0021-8979(00)06703-7]
\end{abstract}

\section{INTRODUCTION}

Electron cyclotron resonance (ECR) plasma sources have extensively been applied to etching and deposition processes in semiconductor manufacture because of their high density and ionization degree with low gas pressure. ${ }^{1,2}$ In these applications, the control of ion behavior is crucial in optimizing the performance of plasma processing such as etching anisotropy, film properties, and surface damage. The ion energy, velocity distribution functions, temperature, and ion current density have been measured in detail. ${ }^{3-11}$ For example, Okuno et al. ${ }^{4}$ reported a detailed measurement of two-dimensional ion velocity distribution functions (IVDFs) and ion temperatures using a directional analyzer in an ECR system. Samukawa et al. ${ }^{5}$ have investigated the dependence of ion current density on the position, magnetic field distribution, and microwave window size. These measurements are very important for the better understanding of the ion properties both in the source area and downstream. On the other hand, numerical modeling has been extensively used to simulate various effects in the plasma. ${ }^{12-17}$ For instance, Hussein et al. ${ }^{12}$ studied the effect of collisions on ion dynamics in the downstream region using a one-dimensional kinetic model. Zhong and co-workers ${ }^{13}$ simulated downstream ion transport by a Monte Carlo method. Porteous and colleagues ${ }^{14}$ investigated the dependence of ion energy and impact angle distribution versus radial position at the substrate surface with a two-dimensional (2D) hybrid model. In our previous articles, ${ }^{16,17}$ using the hybrid model presented in Ref. 14, we reported the variation of the IVDFs in the downstream region of a compact ECR reactor. Recently, some improvements such as the self-consistent treatment of neutral

\footnotetext{
${ }^{a)}$ Present address: Institute of Plasma Physics, Chinese Academy of Sciences, Hefei, Anhui, 230031, People's Republic of China; electronic mail: mhliu@mail.ipp.ac.cn
}

particle density and the inclusion of the influence of electronion collision have been taking into consideration by $\mathrm{Wu}^{15}$ and Cronrath, ${ }^{18}$ respectively. In these previous publications, ${ }^{14-18}$ the microwave absorption is always assumed to be a fixed function of the magnetic field and does not depend on any plasma parameters. But in real experiments, the microwave absorption is strongly dependent on the local electron density and temperature, etc. In this article, an important improvement was made to the previous physical mode ${ }^{14}$ by treating self-consistently the microwave power absorption at the resonant region. With the refined model, the evolution of the IVDFs from source area to the downstream region and the ion current density at the substrate surface in an extended ECR system were investigated.

The article is organized as follows: a brief review of the design features of the ECR reactor and the improved physical model are presented in Sec. II, followed by discussions of the simulation results in Sec. III. The conclusions are summarized in Sec. IV.

\section{THE DESIGN FEATURES AND PHYSICAL MODEL}

\section{A. The design features of the ECR reactor}

The ECR reactor in this simulation is identical to that described by Zhan. ${ }^{19}$ Microwaves $(2.45 \mathrm{GHz})$ were supplied to the cylinder vacuum chamber $(16 \mathrm{~cm}$ in diameter, $33 \mathrm{~cm}$ in length) through a quartz window $(10 \mathrm{~cm}$ in diameter, 18 $\mathrm{cm}$ in length). The divergent magnetic field was established by two external coils. The axial position of the resonant zone is about $9-13 \mathrm{~cm}$. The substrate sites are on the bottom of the chamber. The reactor walls are grounded except the microwave dielectric window. Argon is used as the working gas. 


\section{B. Physical model}

Our physical model is a fluid electron-particle ion hybrid model in 2D cylindrical axisymmetric geometry. Its characteristics have been described in detail elsewhere, ${ }^{14-17}$ so we only outline its major features as follow.

The electron behaviors are described by the steady fluid equations with mass, momentum, and energy conservation:

$$
\begin{aligned}
& \nabla \cdot \mathbf{j}_{e}=R_{\mathrm{ion}}\left(n_{e}, n_{i}, n_{0}\right) ; \\
& \mathbf{j}_{e}=\mu\left[n_{e} \nabla \phi-\nabla\left(n_{e} T_{e}\right) / e\right], \\
& \mathbf{Q}=2 k T_{e}\left(\mathbf{j}_{e}-\mu n_{e} \nabla T_{e} / e\right), \\
& \nabla \cdot \mathbf{Q}=P_{\mathrm{ecr}}+e \mathbf{j}_{e} \cdot \nabla \phi-P_{\text {loss }} .
\end{aligned}
$$

The set of equations is closed with Poisson's equation:

$$
\varepsilon_{0} \Delta \phi=e\left(n_{e}-n_{i}\right)
$$

Here $n_{e}, n_{i}, n_{0}, \phi, T_{e}, \mathbf{j}_{e}, \mathbf{Q}, \mu, R_{\text {ion }}$, and $\varepsilon_{0}$ are electron density, ion density, neutral density, electrostatic potential, electron temperature, electron flux, electron energy flux, mobility, ionization rate, and permeability, respectively. $P_{\text {loss }}$ and $P_{\text {ecr }}$ are the power consumption for various loss mechanisms and the microwave power absorption, respectively.

Ions are treated as individual particles and their dynamic behaviors are modeled by combining the particle-in-cell and the Monte Carlo method in two spatial dimensions $(r, z)$ and three velocity space dimensions $\left(v_{r}, v_{\theta}, v_{z}\right)$. The sheath on the boundary is assumed to be infinitely thin and is treated analytically.

The detailed description of the self-consistent microwave power absorption is not the main topic in this article, but can be found in Ref. 20. Here we only give the final expression of the microwave power absorption in the resonance zone:

$$
\begin{aligned}
P_{\mathrm{ecr}}= & \operatorname{Im}\left\{2 \frac{\omega_{p}^{2}}{k_{\|}^{2} \boldsymbol{v}_{T}^{2}} \varepsilon_{0}\left[1+\frac{\omega}{k_{\|} \boldsymbol{v}_{T}} Z_{p}\left(\frac{\omega}{k_{\|} \boldsymbol{v}_{T}}\right)\right]\right\}\left|E_{z}\right|^{2} \\
& \left.+\operatorname{Im}\left\{\sqrt{2} \varepsilon_{0} \frac{\omega_{p}^{2}}{k_{\|}^{-} \boldsymbol{v}_{T}} Z_{p}\left(\frac{\omega+\omega_{c}}{k_{\|}^{-} \boldsymbol{v}_{T}}\right)\right]\right\}\left|E_{-}\right|^{2} \\
& \left.+\operatorname{Im}\left\{\sqrt{2} \varepsilon_{0} \frac{\omega_{p}^{2}}{k_{\|}^{+} \boldsymbol{v}_{T}} Z_{p}\left(\frac{\omega-\omega_{c}}{k_{\|}^{+}}\right)\right]\right\}\left|E_{+}\right|^{2} .
\end{aligned}
$$

The terms on the right hand side represents the contribution of the Landau damping, the anomalous Doppler damping, and the cyclotron damping sequentially. Here $\varepsilon_{0}, v_{T}, \omega, \omega_{p}, \omega_{c}$ are the permeability, thermal velocity of the electrons, microwave frequency, plasma frequency, and electron cyclotron resonance frequency, respectively. $E_{z}$ is the axial component of the microwave electric field, $E_{-}$and $E_{+}$are the electric field components of the left and right hand polarized wave respectively, $k_{\|}, k_{\|}^{-}$, and $k_{\|}^{+}$are the corresponding wave vectors. So $P_{\text {ecr }}$ in Eq. (6) is directly related to plasma parameters such as plasma density, electron temperature, magnetic field profile, etc.

\section{Microwave field analysis in the resonant cavity}

In order to illustrate the effects of the microwave mode on the plasma parameters, it is necessary to study the propagation of the electromagnetic waves in the resonant cavity.

The axial component of $E_{z}$ and $H_{z}$ can be obtained from Maxwell's equations in the plasma. ${ }^{21}$

$$
\begin{aligned}
& \nabla_{\perp}^{2} H_{z}+d H_{z}=g E_{z}, \\
& \nabla_{\perp}^{2} E_{z}+a E_{z}=b H_{z} .
\end{aligned}
$$

For the transverse electric (TE) mode, $E_{z}=0, d=k_{0}^{2} \varepsilon_{1}-k_{z}^{2}$ $+k_{0}^{2} \varepsilon_{2}^{2} / \varepsilon_{1}$, and the symmetric solution of Eq. (7) is

$$
\begin{aligned}
B_{z}= & B_{z}^{0}\left(A\left\{\begin{array}{l}
J_{0}(\alpha r) \\
I_{0}(p r)
\end{array}\right\}+i B\left\{\begin{array}{c}
J_{0}(\beta r) \\
I_{0}(q r)
\end{array}\right\}\right) e^{-k_{i} z} e^{i\left(k_{r} z-\omega t\right)}, \\
B_{r}= & -i\left[\left(k_{\|}^{2}-\frac{\omega^{2}}{c^{2}} \varepsilon_{\perp}\right)^{2}+\left(\frac{\omega^{2}}{c^{2}} \varepsilon_{x}\right)^{2}\right]^{-1} \\
& \times\left[k_{\|}\left(k_{\|}^{2}-\frac{\omega^{2}}{c^{2}} \varepsilon_{\perp}\right) \partial_{r} B_{z}\right], \\
B_{\theta}= & -i\left[\left(k_{\|}^{2}-\frac{\omega^{2}}{c^{2}} \varepsilon_{\perp}\right)^{2}+\left(\frac{\omega^{2}}{c^{2}} \varepsilon_{x}\right)^{2}\right]^{-1}\left[k_{\|} \frac{\omega^{2}}{c^{2}} \varepsilon_{x} \partial_{r} B_{z}\right], \\
E_{r}= & \frac{\omega}{k_{\|}} B_{\theta}, E_{\theta}=-\frac{\omega}{k_{\|}} B_{r} .
\end{aligned}
$$

For the transverse magnetic (TM) mode, $H_{z}=0$, $a=-k_{z}^{2} \varepsilon_{3} / \varepsilon_{1}+k_{0}^{2} \varepsilon_{3}$, and the solution of Eq. (8) is

$$
\begin{aligned}
E_{z}= & E_{z}^{0}\left(C\left\{\begin{array}{c}
J_{0}(\alpha r) \\
I_{0}(p r)
\end{array}\right\}+i D\left\{\begin{array}{c}
J_{0}(\beta r) \\
I_{0}(q r)
\end{array}\right\}\right) e^{-k_{i} z} e^{i\left(k_{r} z-\omega t\right)}, \\
E_{r}= & -i\left[\left(k_{\|}^{2}-\frac{\omega^{2}}{c^{2}} \varepsilon_{\perp}\right)^{2}+\left(\frac{\omega^{2}}{c^{2}} \varepsilon_{x}\right)^{2}\right]^{-1} \\
& \times\left[k_{\|}\left(k_{\|}^{2}-\frac{\omega^{2}}{c^{2}} \varepsilon_{\perp}\right) \partial_{r} E_{z}\right], \\
E_{\theta}= & -i\left[\left(k_{\|}^{2}-\frac{\omega^{2}}{c^{2}} \varepsilon_{\perp}\right)^{2}+\left(\frac{\omega^{2}}{c^{2}} \varepsilon_{x}\right)^{2}\right]^{-1}\left[k_{\|} \frac{\omega^{2}}{c^{2}} \varepsilon_{x} \partial_{r} E_{z}\right],
\end{aligned}
$$

where $k_{0}^{2}=\omega^{2} / c^{2}, \varepsilon_{1}, \varepsilon_{2}, \varepsilon_{3}\left(\varepsilon_{\perp}, \varepsilon_{x}, \varepsilon_{\|}\right)$are the elements of the electric tensor $\overrightarrow{\boldsymbol{\varepsilon}}$ and $A, B, C$, and $D$ are complex constants. The values of $\alpha, \beta, p, q$ and the choice of Bessel functions are detrmined by the value of $a, d$, and the boundary conditions.

\section{RESULTS AND DISCUSSION}

The main purpose of this article is to show the ion transport properties both in the source area and downstream region. For an extended ECR plasma reactor, the IVDFs in the source area which were excluded in a compact ECR system $^{16,17}$ are especially of concern. In order to analyze the simulation results, the variations of the ion density and plasma potential with position are shown in Figs. 1 and 2, respectively. The two profiles are similar, they extend maxima at $z \sim 5 \mathrm{~cm}, r=0 \mathrm{~cm}$, then decrease towards the microwave window (upstream), the bottom (downstream), and the cylindrical wall. 


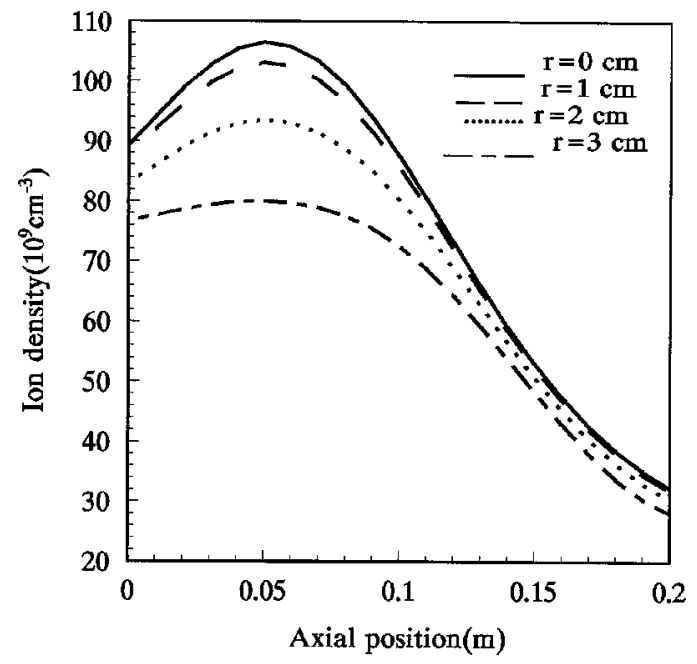

FIG. 1. The dependence of ion density vs axial position at different radial positions. power $=450 \mathrm{~W}$, pressure $=0.6 \mathrm{mTorr}$ (TM mode).

\section{A. Ion velocity distribution in the source area and downstream}

We collect and analyze the ion velocity distributions at several grids $(1 \times 1 \mathrm{~cm})$ for different values of $z$. For the following graphs, the gas pressure is $0.6 \mathrm{mTorr}$, the microwave is TM mode and its power is $450 \mathrm{~W}$.

Figure 3 shows the axial velocity distribution of ions in the source area. At the resonant region $(9<z<10 \mathrm{~cm})$, the IVDF is a bimodal distribution. Towards the microwave window, the IVDF becomes a narrower single-peak distribution with a lower average value. Downstream, the IVDF is a broader distribution. These tendencies can be attributed to the variation of the plasma electrostatic field shown in Fig. 2. In addition, the width of these distribution functions increases slightly with the radial position.

The radial and azimuthal ion velocity distribution for several axial positions in the source area is given in Figs. 4 and 5, respectively. These distribution functions become narrower as the axial positions increase. On the other hand, both

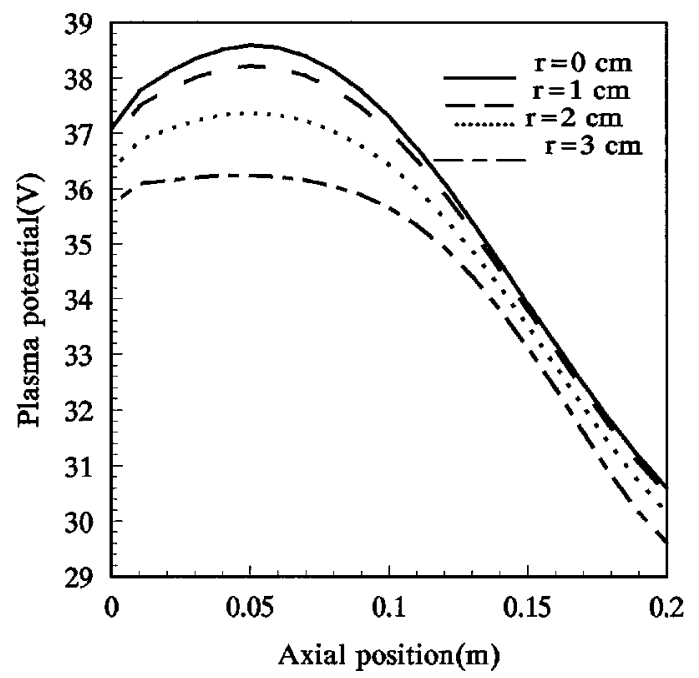

FIG. 2. The variation of plasma potential with axial position at different radial positions. The operating conditions are the same as in Fig. 1.

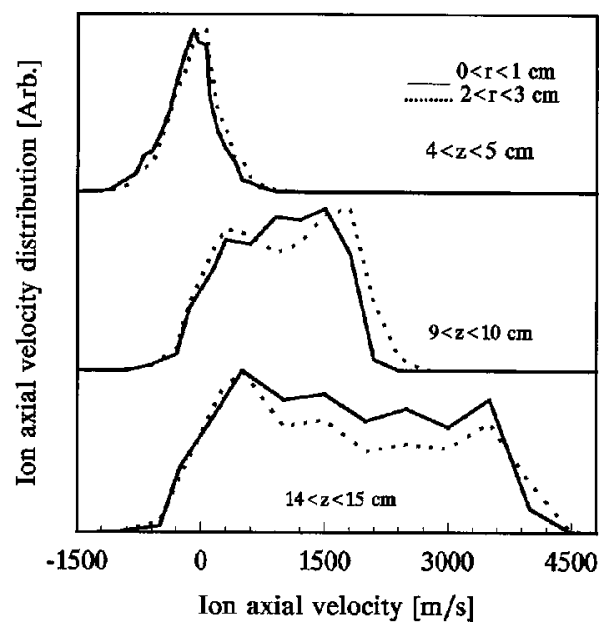

FIG. 3. The axial velocity distribution of ion at different positions in the source area. The operating conditions are shown in Fig. 1.

the average velocity and the full width of half maximum (FWHM) of these distribution increase with radial position. These results can be interpreted using the data in Figs. 1 and 2: the radial electrostatic field and the ion density gradient are the decreasing functions of the axial position, while the axial electrostatic field and the ion density gradient have the opposite tendency. A similar, but more detailed analysis can be found in Ref. 16.

Figure 6 illustrates the downstream axial velocity distribution of ions $f\left(v_{z}\right)$. It is obvious that the IVDF is bimodal. This result is qualitatively consistent with many experimental observations. ${ }^{3,19}$ As the axial position increases, $f\left(v_{z}\right)$ shifts to the high velocity region due to the acceleration by the axial electrostatic field, meanwhile the average velocity of these distribution decreases with increasing radial position.

It can be seen obviously from Figs. 3 and 6 that the IVDF of source area is distinctly different from the downstream IVDF. The bimodal IVDFs downstream are not only contributed to the distributed ionization, charge exchange,

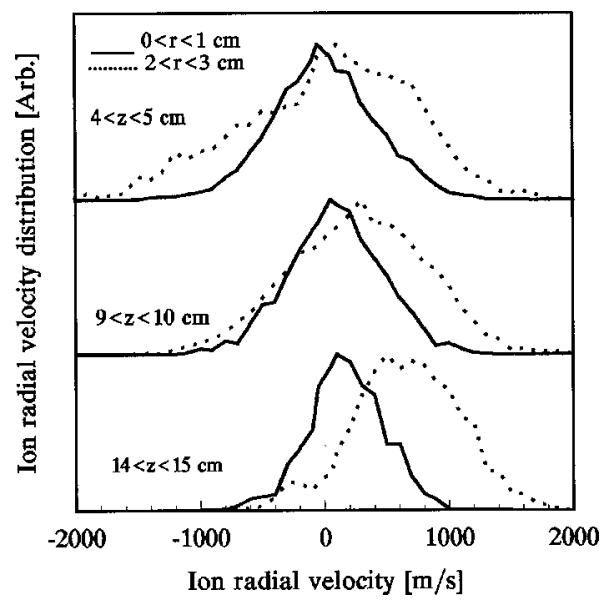

FIG. 4. The radial velocity distribution of ion at different positions in the source area. The operating conditions are given in Fig. 1. 


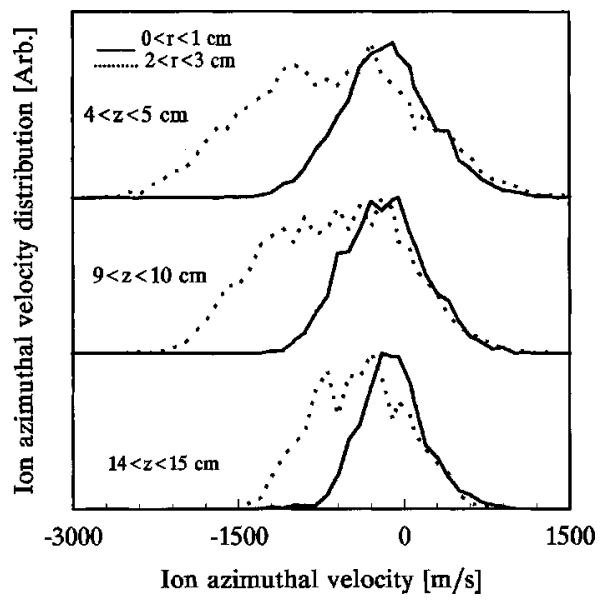

FIG. 5. The azimuthal velocity distribution of ion at different positions in the source area. The operating conditions are the same as in Fig. 1.

and the plasma potential drop from source area to downstream region, ${ }^{3}$ but also affected by the IVDFs in the source area (especially at low pressure).

\section{B. Ion angular distribution in the source}

The ion angular distribution function (IADF) is a key parameter for understanding the performance of plasma processing such as etching anisotropy, film properties, and surface damage. The ion radial pitch angle $\left[\operatorname{arctg}\left(v_{r} / v_{z}\right)\right]$ distributions for several positions in the source area are shown in Fig. 7. These distributions become narrower as the axial coordinates increase. This tendency is similar to the corresponding radial IVDF. Meanwhile the small angle part of these distributions becomes dominant with the increasing axial coordinates. Another important indication in Fig. 7 is that the average radial angle shifts to higher values and the width of these distribution increases as the radial coordinate increases. There is also a similar tendency for the ion azimuthal pitch angle distributions.

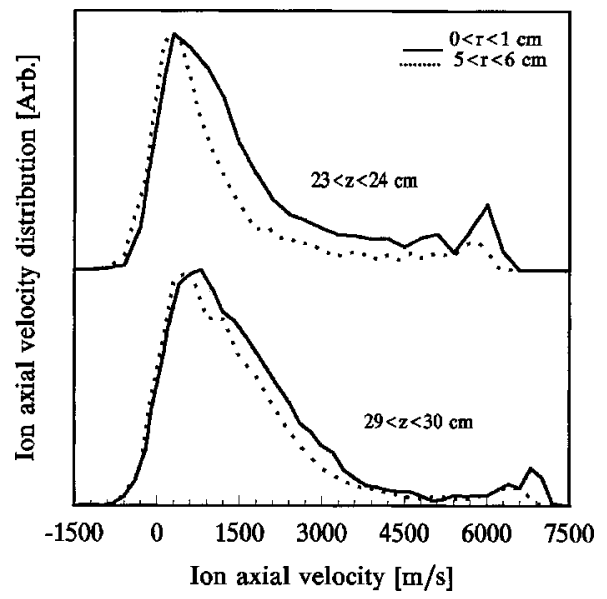

FIG. 6. The ion axial velocity distribution at different positions downstream. The operating conditions are shown in Fig. 1.

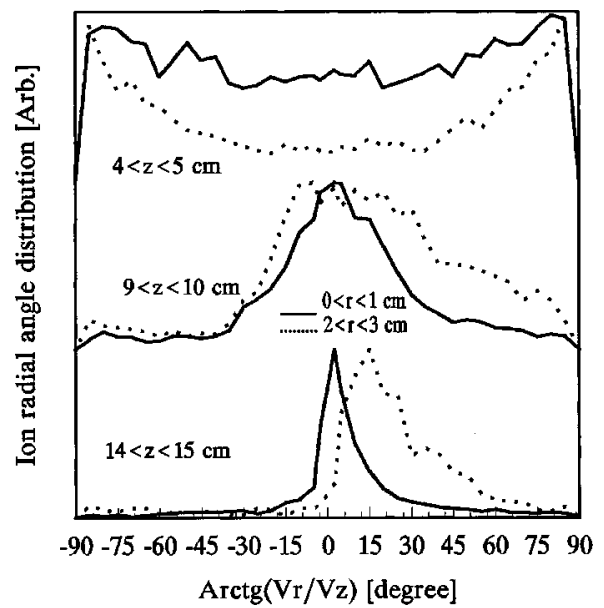

FIG. 7. The ion radial angle distribution at different positions in the source area. The operating conditions are shown in Fig. 1.

\section{Ion velocity distribution versus downstream pressure}

The IVDFs are affected by many factors such as magnetic configuration, microwave mode, operating condition, etc. Figure 8 illustrates the variation of the axial IVDF in the TE mode with pressure. At high pressure, the IVDFs have a symmetric single peak. As the pressure decreases, the tail of the IVDFs rises continuously. At still lower pressure, the IVDF becomes bimodal. This trend is qualitatively consistent with Okuno's experimental measurement. ${ }^{4}$

\section{Ion current density at the substrate surface}

The ion current density and its uniformity at the substrate are important in determining the performance of plasma processing. Ion current density towards the substrate surface can be estimated through the approximate expression ${ }^{9} J \approx e n_{w} c_{s}$, where $n_{w}$ is ion density near the wafer and $c_{s}=\left(k T_{e_{w}} / M_{i}\right)^{0.5}$ is the Bohm velocity. The variation of ion current density in the TM mode with pressure is given in Fig. 9. It can be seen that the ion current densities increase with pressure in the low-pressure region but de-

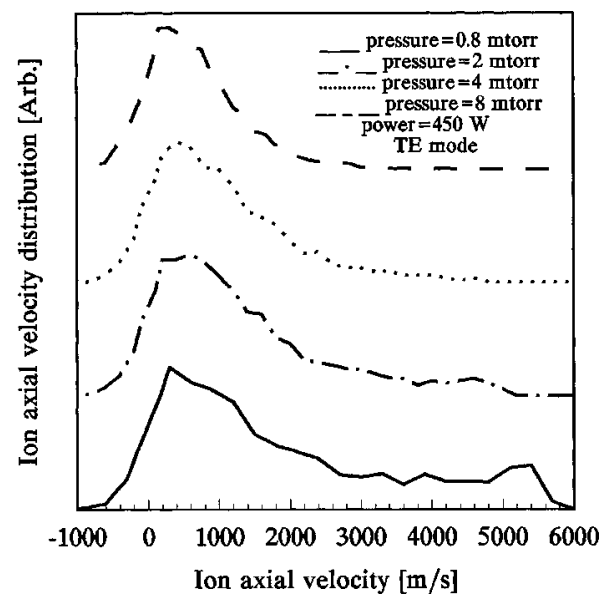

FIG. 8. The ion axial velocity distribution of TE mode for different values of the downstream pressure. $21<z<22 \mathrm{~cm}, 0<r<1 \mathrm{~cm}$. 


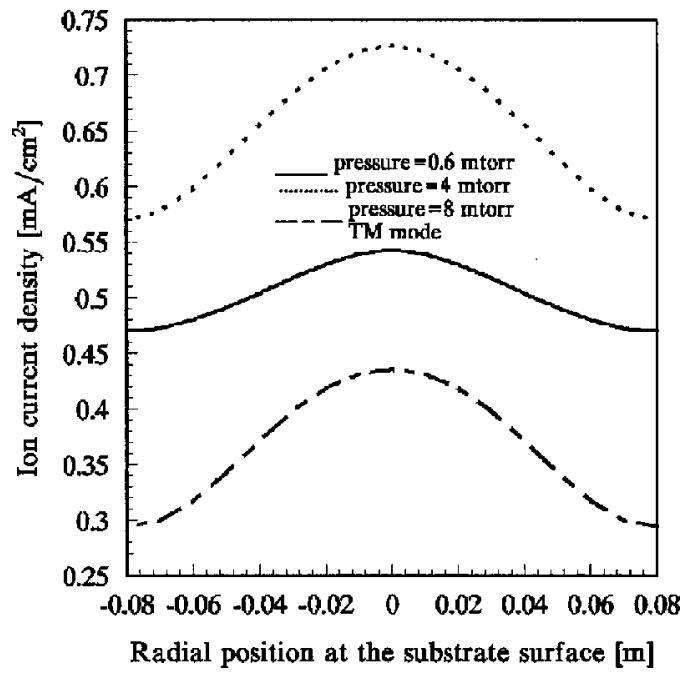

FIG. 9. The ion current density of TM mode vs radial position for different values of pressure at the substrate surface. $32<z<33 \mathrm{~cm}$, power $=450 \mathrm{~W}$.

crease greatly in the high-pressure region. On the other hand, the nonuniformity of the ion current density increases gradually with pressure. These features indicate that the choice of pressure is very important for obtaining high processing rate with good uniformity. The radial distribution of ion current density in the TE mode is plotted in Fig. 10 for different values of the microwave power at the substrate surface. The ion current density increase with microwave power, but its uniformity becomes poorer. These simulation results are qualitatively in good agreement with many experimental observatiaons. ${ }^{5,9-11}$

\section{CONCLUSION}

With a refined 2D hybrid code, we have systematically studied the ion velocity and angle distributions both in the source area and downstream region, and the ion current density at the substrate surface. The dependence of these distri-

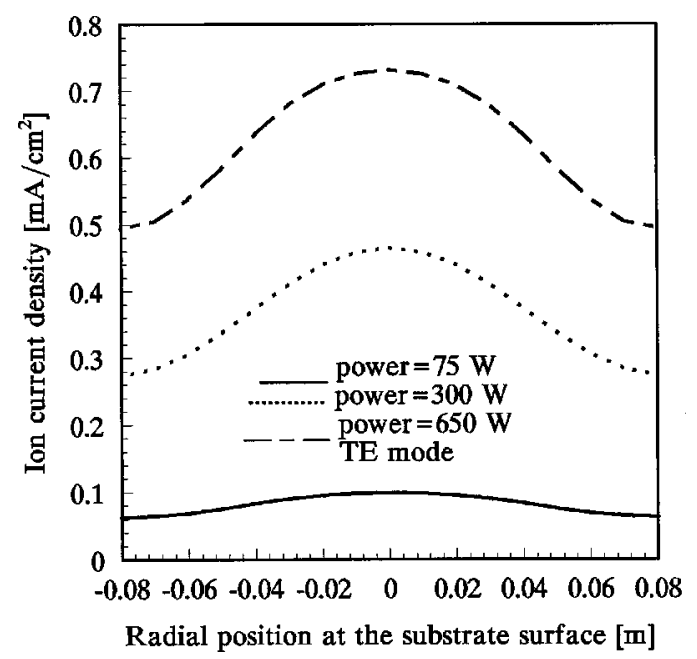

FIG. 10. The ion current density of TE mode vs radial position for different values of the microwave power at the substrate surface. $32<z<33 \mathrm{~cm}$, pressure $=8$ mTorr. bution functions versus the radial position, axial position, pressure, and microwave power are discussed. The main conclusions can be drawn as follows:

(1) Near the microwave window, the axial IVDF has singlepeak symmetric shape with lower average value and narrower width. At the resonant region, the axial IVDF is bimodal. As the axial coordinate increases, the IVDFs become broader. All the widths of these distributions increase with radial coordinate.

(2) The radial and azimuthal IVDFs in the source area become narrower when the axial coordinate increases. Both the average velocity and the FWHM of these distributions increase with increasing radial coordinate.

(3) The bimodal IVDF in the downstream becomes broader as the axial coordinate increases and the average velocity decreases with increasing radial coordinate.

(4) Both the radial and azimuthal pitch angles in the source area shift to higher values and the FWHMs of these distributions increase with the radial coordinate. The small angle part of these distributions becomes dominant as the axial coordinate increases.

(5) As pressure decreases, the shape of the axial IVDF changes gradually from symmetric single peak to bimodal.

(6) The ion current density at the substrate surface increases with pressure in the low-pressure region and decreases greatly in the high-pressure region, but its nonuniformity increases continuously with pressure. The ion current density increases with microwave power, at the same time, its uniformity becomes poorer.

\section{ACKNOWLEDGMENTS}

This work was supported by the National Nature Science Foundation of China and by the Postdoctoral Science Foundation of China. The first author is grateful to Dr. B. M. Harvey for his friendly discussion about the microwave power absorption. Appreciation is also expressed to Professor Y. Y. Li, J. X. Ma, and R. J. Zhan for their enlightening discussions and useful advice.

${ }^{1}$ J. Asmussen, J. Vac. Sci. Technol. A 7, 883 (1989).

${ }^{2}$ Z. Y. Ning and Z. X. Ren, Prog. Phys. 12, 38 (1992) (in Chinese).

${ }^{3}$ N. Sadeghi, T. Nakano, D. J. Trevor, and R. A. Gottscho, J. Appl. Phys. 70, 2552 (1991).

${ }^{4}$ Y. Okuno, Y. Ohtsu, and H. Fujita, J. Appl. Phys. 74, 5990 (1991).

${ }^{5}$ S. Samukawa, S. Mori, and M. Sasaki, J. Vac. Sci. Technol. A 9, 85 (1991).

${ }^{6}$ W. M. Holber and J. Forster, J. Vac. Sci. Technol. A 8, 3720 (1990).

${ }^{7}$ M. Matsuoka and K. Ono, J. Vac. Sci. Technol. A 6, 25 (1988).

${ }^{8}$ E. A. Den Hartog, H. Persing, and R. C. Woods, Appl. Phys. Lett. 57, 661 (1990)

${ }^{9}$ J. L. Cecchi, J. E. Stevens, R. L. Jarecki, and Y. C. Huang, J. Vac. Sci. Technol. B 9, 318 (1991).

${ }^{10}$ S. Samukawa, Y. Nakagawa, and K. Ikeda, Jpn. J. Appl. Phys. 30, 423 (1991).

${ }^{11}$ O. A. Popov, W. Hale, and A. O. Westner, Rev. Sci. Instrum. 61, 300 (1990).

${ }^{12}$ M. A. Hussein, G. A. Emmert, N. Hershkowitz, and R. C. Woods, J. Appl. Phys. 72, 1720 (1991). 
${ }^{13}$ X. X. Zhong, J. D. Wu, C. Z. Wu, and F. M. Li, J. Appl. Phys. 83, 5069 (1998).

${ }^{14}$ P. K. Porteous, H. M. Wu, and D. B. Graves, Plasma Sources Sci. Technol. 3, 25 (1994).

${ }^{15}$ H. M. Wu, D. B. Graves, and M. Kilgore, Plasma Sources Sci. Technol. 6, 231 (1997)

${ }^{16}$ M. H. Liu, X. W. Hu, and H. M. Wu, J. Appl. Phys. 81, 7734 (1997).

${ }^{17}$ M. H. Liu, X. W. Hu, and H. M. Wu, 13th International Symposium on Plasma Chemistry, Beijing, China, 1997, Vol. 2, p. 662.
${ }^{18}$ W. Cronrath, N. Mayumi, M. D. Bowden, K. Uchino, and K. Muraoka, J. Appl. Phys. 82, 1036 (1997).

${ }^{19}$ R. J. Zhan, X. Y. Wang, X. H. Wen, and J. Y. Liu, 2nd Asia-Pacific Conference on Plasma Science and Technology, Sept. 1994, Daejeon, Korea (unpublished).

${ }^{20}$ M. H. Liu, Doctoral dissertation, University Science Technology of China, Oct. 1997 (in Chinese) (unpublished); M. H. Liu, X. W. Hu, Q. C. Wu, and G. Y. Yu, Chin. Phys. Lett. 17(1), 31 (2000).

${ }^{21}$ W. P. Allis, S. J. Buchsbaum, and A. Bers, Wave in Anisotropic Plasma (MIT, Cambridge, MA, 1963). 